\title{
Characterization of Mild Whole-Body Hyperthermia Protocols Using Human Breast, Ovarian, and Colon Tumors Grown in Severe Combined Immunodeficient Mice
}

\author{
E.A. Repasky, ${ }^{1 *}$ E. Tims, ${ }^{1}$ M. Pritchard, ${ }^{1}$ and R. Burd ${ }^{2}$ \\ ${ }^{1}$ Department of Immunology, Roswell Park Cancer Institute, Buffalo, NY \\ ${ }^{2}$ Division of Radiation Oncology, Thomas Jefferson University Hospital, Philadelphia, PA
}

\begin{abstract}
Objective: We have shown that one treatment of fever-like whole body hyperthermia (WBH) on mice bearing human breast tumors results in a tumor growth delay. Our goal was to repeat this study in mice bearing human ovarian or colon tumors. We further evaluated this WBH protocol by performing multiple and interrupted WBH treatments.

Methods: Human tumors were grown in severe combined immunodeficient (SCID) mice. For WBH, core body temperatures were maintained at $39.8 \pm 0.2^{\circ} \mathrm{C}$ for $6-8$ hours. Multiple treatments were given 6-7 days apart. Interrupted WBH consisted of three 2-hour heatings, 15 minutes apart. Tumor growth time (TGT) was the number of days to grow 1.5 or 2 times in volume.

Results: For WBH-treated ovarian tumors, TGT was $12 \pm 1.2 \mathrm{~d}$, compared with $5.0 \pm 0.1 \mathrm{~d}$ for untreated mice $(P<0.05)$. For colon tumors with one WBH treatment TGT was $4.4 \pm 1.1 d$. Two and three treatments had TGTs of $9 \pm 2.3 \mathrm{~d}$ and $8 \pm 1.6 \mathrm{~d}$. For the untreated tumors, TGT was $2 \pm 0.7 \mathrm{~d}$ $(P<0.01$ for one, two, and three treatments). Histological examination indicated that one and two treatments were associated with cellular damage within the tumors. With a slower growing colon tumor, the TGT was $24 \pm 3.3 \mathrm{~d}$ with three WBH treatments, compared with $14 \pm 1.8 \mathrm{~d}$ for controls $(P<0.01)$. The TGT of breast tumors treated with interrupted WBH was not significantly different than the noninterrupted, with TGT of $7.3 \pm 0.8 \mathrm{~d}$ and $6.2 \pm 1.0 \mathrm{~d}$, respectively.

Conclusions: These data illustrate that WBH causes a tumor growth delay in mice bearing human ovarian and colon tumors. This response is enhanced with a second treatment of WBH. Interrupted and noninterrupted WBH give comparable anti-tumor results. We will continue to evaluate WBH in various animal models to optimize its potential for clinical administration and maximize the anti-tumor response. Infect. Dis. Obstet. Gynecol. 7:91-97, 1999. ( 1999 Wiley-Liss, Inc.
\end{abstract}

\section{KEY WORDS}

hyperthermia; breast tumors; ovarian tumors; immune response; heat shock proteins

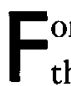
or many solid tumors, it is possible to control the initial malignancy with surgical excision. Unfortunately, surgical methods are not as successful if the cancer recurs or spreads to other sites in the body. Although there are already systematic treatments, such as chemotherapy, applied to most patients as follow up to surgery, it is evident that only a small fraction of patients actually benefit

Grant sponsor: National Institute of Health; Grant No: CA71599. Grant Sponsor: Department of Defense; Grant Nos: DAMD 17-98-1-8104 and 17-98-1-8311.

*Correspondence to: Elizabeth A. Repasky, PhD, Department of Immunology, Roswell Park Cancer Institute, Buffalo, NY 14263. 
from this treatment in terms of overall survival. The goal of our laboratory is to develop better therapies to enhance a patient's own immune response against his or her tumor; these therapies may be useful alone or as an adjuvant therapy in combination with chemotherapy or radiation. A large part of our effort is being devoted to the examination of mild, long-duration (fever-like) whole-body hyperthermia $(\mathrm{WBH})$ as a possible means to help stimulate the immune system. ${ }^{1-3}$

When clinical hyperthermia was first conceived for use in cancer treatment, it was envisioned as a type of thermal radiation therapy whereby a heat treatment sufficient to sterilize or directly kill cancer cells would selectively be delivered to the tumor. It has been noted in the past few years, however, that the tumor temperatures achieved during these local treatments were well below those known to be cytotoxic. ${ }^{4-6}$ Whole-body hyperthermia protocols have been developed that have employed temperatures as high as $42^{\circ} \mathrm{C}$ given for durations ranging from $15 \mathrm{~min}$. to 2 hours. ${ }^{7-10}$ For the studies described herein, we utilized a mild (feverlike) WBH protocol in which the core body temperature is maintained at $39.8^{\circ} \mathrm{C}$ for $6-8$ hours. ${ }^{1,3}$ This is similar to that obtained during a normal febrile episode, and other work in our lab is devoted to determining what immunological effector activity is affected by the thermal element of fever. $^{2,11,12}$

We have previously shown that a single treatment of mild WBH caused a significant (albeit temporary) reduction of tumor volume and a tumor growth delay in severe combined immunodeficient (SCID) mice bearing human breast xenografts and Balb/c mice bearing transplantable, syngeneic tumors. ${ }^{1}$ The anti-tumor effect seen with the treatment was correlated with the appearance of large numbers of apoptotic tumor cells and an increase in the size of intratumor blood vessels. Substantial levels of several heat shock proteins (Hsps), including Hsp70 and Hsp110, are also induced in the tumor. ${ }^{12}$ Increased numbers of granulocytes, macrophages, and lymphocytes were also observed in the tumor vasculature and in the tumor stroma immediately following WBH exposure. Our laboratory has also shown that WBH results in several changes in tissue lymphocytes indicative of altered activation levels. These include the alterations in cellular and subcellular proteins, adhesion, and the induction of Hsps. ${ }^{2,11,12}$ These data provide evidence that there may in fact be an enhancement of the immune system with mild WBH. It is likely that these alterations in structure and function of immune cells that we have recognized are associated with the tumor killing we have observed.

In this paper, we describe experiments to further characterize this fever-like WBH protocol. We have expanded our studies to include human ovarian and colon tumor xenografts. We have investigated the potential to enhance the observed antitumor response with additional treatments of WBH. Lastly, we have altered the protocol to give WBH in two short increments, instead of one long treatment, so that it may be more clinically tolerable.

\section{SUBJECTS AND METHODS}

\section{Tumor Implantation and Growth}

Human ovarian (\#8436), colon (\#9427), and breast (\#8600) tumor surgical specimens were received from the Roswell Park Cancer Institute Tissue Procurement Facility (Buffalo, NY). These tissues were implanted subcutaneously into 8-week old female c.b.17 scid/scid mice from Taconic Laboratories (Germantown, PA). These tumors were passaged several times in SCID mice and dissected into pieces $2 \mathrm{~mm}$ in diameter, which were implanted subcutaneously into SCID mice for use in these experiments. After the tumors reached a volume of approximately $60 \mathrm{~mm}$, WBH was initiated. ${ }^{3}$ The tumors were measured with a vernier caliper every 2 days to determine the shortest diameter (A) and the longest diameter (B). The volume was then calculated by using the formula $V=\left(A^{2} B\right) / 2$. The relative tumor growth $\left(\mathrm{T}_{\mathrm{r}}\right)$ was then calculated for each tumor by dividing the volume at any given day after WBH $\left(\mathrm{T}_{\mathrm{d}}\right)$ by the volume at the start of WBH $\left(\mathrm{T}_{0}\right)$.

\section{WBH Treatment}

Mice were placed in microisolater cages preheated to $39^{\circ} \mathrm{C}$ that contained food, bedding, and water; it is important that the water was at least $39^{\circ} \mathrm{C}$. The cages were then placed in a gravity convection oven (Memmert model BE500, East Troy, WI) with preheated incoming fresh air. The average body temperature of the mice before WBH was $37.5^{\circ} \mathrm{C}$. The body temperature was gradually in- 


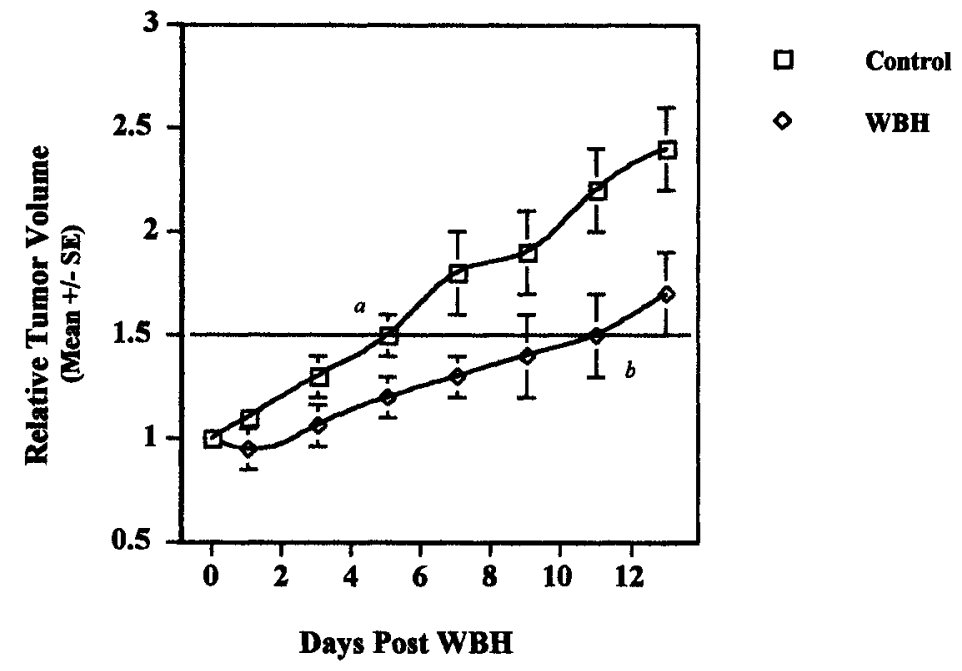

Fig. I. Effect of WBH on the growth of human ovarian tumor \#8436. SCID mice bearing a human ovarian tumor were treated with WBH for 6 hours on day 0 . Tumors were measured with calipers every other day and relative tumor volume was calculated and graphed vs. days post-WBH (see "Materials and Methods"). In the control group (a; $\mathbf{n}=5$ ), TGT was 5.0 $\pm 0.1 \mathrm{~d}$. In the WBH treated group $(\mathrm{b} ; \mathrm{n}=5)$, TGT was $12.0 \pm 1.2 \mathrm{~d}(\mathrm{P}<0.05$ when compared with the control; Student $t$ test).

creased $1^{\circ} \mathrm{C}$ every 30 minutes until a core temperature of $39.8^{\circ} \mathrm{C}\left( \pm 0.2^{\circ} \mathrm{C}\right)$ was achieved. The temperature of the incubator was adjusted when necessary to maintain a constant core temperature of $39.8^{\circ} \mathrm{C}$ for $6-8$ hours. For some experiments, the 6-8 hour heating was repeated three times every 6-7 days. In other experiments, this heating protocol was altered such that the mice were taken out of the incubator after 3 hours of heating at $39.8^{\circ} \mathrm{C}$ and remained at room temperature for 15 minutes. The mice then were returned to the incubator to receive another 3 hours of heating at $39.8^{\circ} \mathrm{C}$. During WBH, the core temperatures of the mice were monitored with the Electric Laboratory Animal Monitoring System Pocket Scanner (model 5007) from Biomedic Data Systems (Maywood, NJ). Microchip transponders $(14 \mathrm{~mm} \times 2.2 \mathrm{~mm}$ ) were subcutaneously implanted into the dorsal thoracic area with a trocar into nonexperimental animals. These animals were placed in cages with their experimental animal counterparts at least 24 hours prior to the initiation of the WBH treatment. 'Temperatures were collected using the pocket scanner, which has a liquid-crystal display screen where the temperature information is displayed. We found that it was not difficult to maintain body temperature between $39^{\circ} \mathrm{C}$ and $40^{\circ} \mathrm{C}$. When heating several animals, internal variability between individual animals was $\pm 0.2^{\circ} \mathrm{C}$.

After the WBH treatment, animals were placed back in their regular cages. In some experiments, a representative animal from each group was sacrificed 8 hours after the WBH treatment to examine the histology of the tumors. Tumor measurements were taken as described above for the remainder of the experiment.

\section{Statistical Analysis of Data}

Tumor growth time (TGT), or time for the tumor volume to reach 1.5 times (for the ovarian and colon tumor experiments) or 2 times (for the breast tumor experiments) its original tumor volume was calculated in days. A Student $t$ test was performed to compare TGT values for $\mathrm{WBH}$ treated mice with those for control mice.

\section{RESULTS}

\section{Effects of WBH on SCID Mice Bearing Human Ovarian Tumors}

Whole-body hyperthermia was effective in exerting an anti-tumor response on SCID mice bearing human ovarian tumors (Fig. 1). The TGT for this experiment was the number of days for the tumor to reach 1.5 times its original volume. The TGT of the unheated control tumors for this experiment was as $5.0 \pm 0.1 \mathrm{~d}$. The TGT of the WBH-treated tumor was $12 \pm 1.2 \mathrm{~d}(P<0.05)$. These results are comparable to our previous data using human breast tumors. 


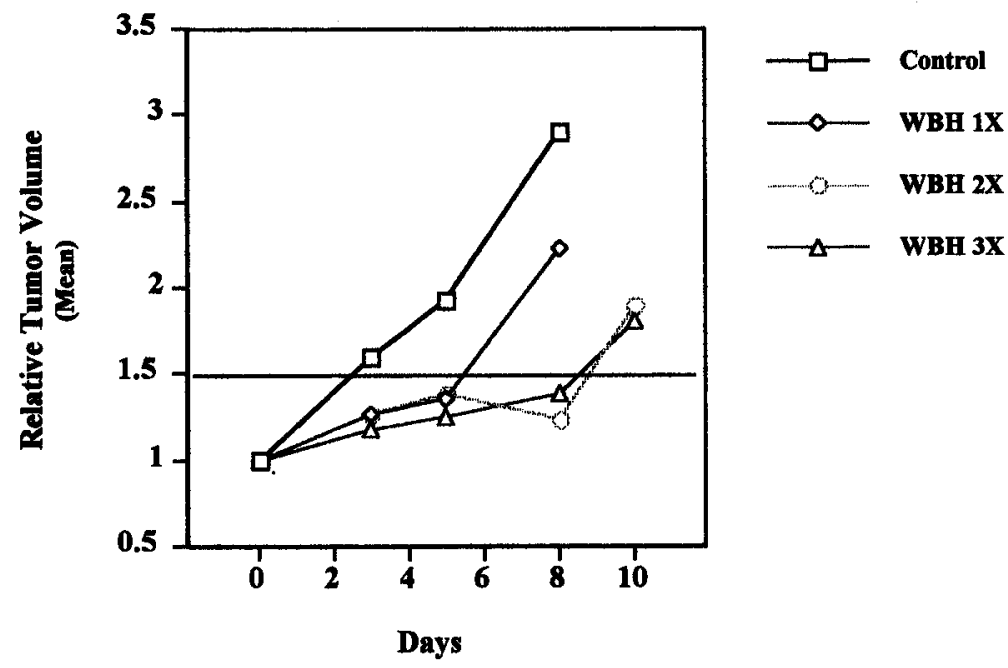

Fig. 2a. Effect of multiple WBH treatments on a fast-growing human colon tumor \#9427. SCID mice bearing a human colon tumor were treated with WBH for 6 hours on days 0,5 , and II. Tumors were measured with calipers regularly for the duration of the experiment. Relative tumor volume was calculated and graphed vs. days. Control, $n=4 ; W B H I x, n=4 ; W B H$ $2 x, n=4$; WBH $3 x, n=4$.

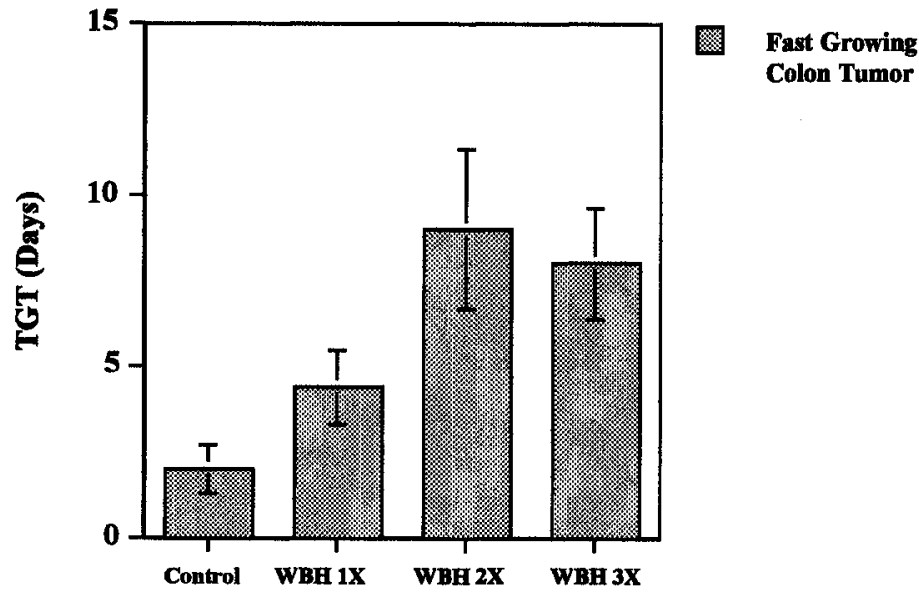

Fig. 2b. Time for tumor to reach $1.5 \times$ original volume (TGT). TGTs were calculated for each treatment group from the mice in Fig. 2a. In mice with colon tumors receiving one WBH treatment, TGT was $4.4 \pm 1.1 \mathrm{~d}$. Those with two and three treatments had TGTs of $9.0 \pm 2.3 \mathrm{~d}$ and $8.0 \pm 1.6$ days. For the untreated tumors, TGT was $2.0 \pm 0.7 \mathrm{~d}(P<0.01$ for one, two, and three treatments when compared with the control; Student $t$ test).

Effects of Single and Multiple Treatments of WBH on Mice Bearing a Fast-Growing and a Slow-Growing Variant of a Human Colon Tumor

To investigate the effects of WBH on fast- and slow-growing tumors, we utilized a colon tumor that has both a fast- and slow-growing variant. The tumors in the mice bearing the fast-growing variant of a human colon tumor responded to WBH (Fig. 2a). The TGT for this tumor was the number of days for the tumor to reach 1.5 times its original volume. The control TGT was $2 \pm 0.7 \mathrm{~d}$. The TGT for the tumors treated one time with WBH was 4.4 $\pm 1.1 \mathrm{~d}(P<0.01)$. Those that were given a second treatment also responded to hyperthermia, and the anti-tumor response was greater (TGT was $9 \pm$ $2.3 \mathrm{~d}, P<0.01$ when compared with the control). For the mice that were given a third treatment, the response was not significantly different when compared with those that received two treatments (TGT was $8 \pm 1.6$ days, $P<0.01$ when compared with control) (Fig. 2b). Histological examination of 


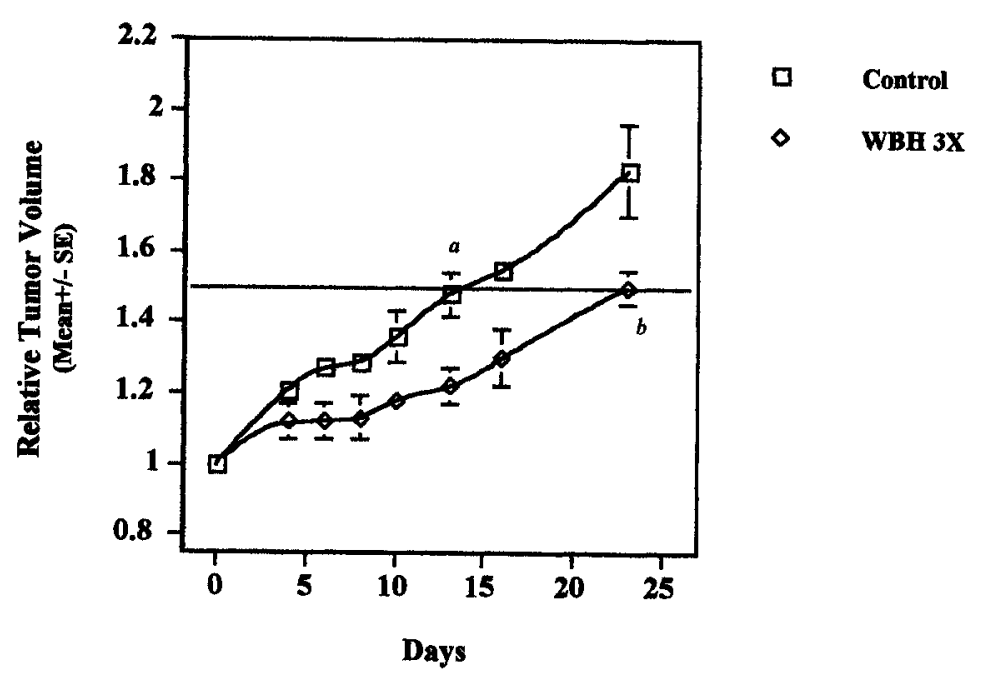

Fig. 3. Effect of multiple WBH treatments on the growth of a slow-growing colon tumor \#9427. SCID mice bearing a human colon tumor were treated with three doses of WBH for 6 hours on days 0,7 , and 14. Tumors were measured with calipers regularly for the duration of the experiment. Relative tumor volume was calculated and graphed vs. days. In the control group $(a ; n=7)$, TGT was $14.3 \pm 1.8 \mathrm{~d}$. In the WBH $3 x$ group $(b ; n=7)$, TGT was $25.0 \pm 3.3 \mathrm{~d}$. $(P<0.01$ when compared with the control).

the tumors indicated that the first and second treatments of hyperthermia were associated with changes indicative of cellular damage; following a third treatment, tumor structure was comparable to the nonheated control (data not shown). The induction of Hsp70 and Hsp110 was also seen in these tumors following WBH (data not shown). The extent of the anti-tumor responses that have been observed differ from tumor to tumor. For example, when tumor growth was evaluated for a slow-growing variant of the same human colon tumor the TGT was $25 \pm 3.3 \mathrm{~d}$. When WBH was administered, the TGT was $14.3 \pm 1.8 \mathrm{~d}, P<0.01$ (Fig. 3). For the fast-growing variant, the time to reach 1.5 times the original volume was increased by $300 \%$ with the treatment. Whole-body hyperthermia on the slow-growing tumor increased the TG'T by $108 \%$.

\section{Effects of Interrupted WBH on SCID Mice Bearing Human Breast Tumors}

Uninterrupted and interrupted WBH treatments were very similar with regard to the tumor growth delay (Fig. 4). Due to the rapid growth rate of this tumor, TGT was the time for tumor volume to double. For the control tumor, the TGT was $3.0 \pm$ $0.1 \mathrm{~d}$. The interrupted and uninterrupted treatments had TGTs of $7.3 \pm 0.8 \mathrm{~d}$ and $6.2 \pm 1.0 \mathrm{~d}$, respectively $(P<0.01, P<0.01$ when compared with control). There was no statistical difference between the interrupted and continual WBH treatments.

\section{DISCUSSION}

We have repeatedly observed that fever-like WBH exerts an anti-tumor effect on the growth of human tumors in SCID mice. Human breast, ovarian, and colon tumors respond to this therapy in a similar manner, indicating that the temporary tumor growth delay is most likely not tumor specific. The extent of this response, however, does show some variation between tumors. This difference could be attributed to inherent differences between tumors. This data is highly suggestive that applications of this mild WBH protocol in a clinical setting may be beneficial to patients. Although the result is temporary, it may be enough to give an advantage to current strategies, such as chemotherapy or radiation therapy, which are not always as effective as one would hope. Also, many of these regimens exert toxic side effects at the high doses necessary for success; perhaps WBH could act as an adjuvant to allow these therapies to be given at less toxic doses that would still be effective. Moreover, since our data indicate that WBH is most likely immunostimulatory, combining hyperthermia with different types of immunotherpy, such as cytokine 


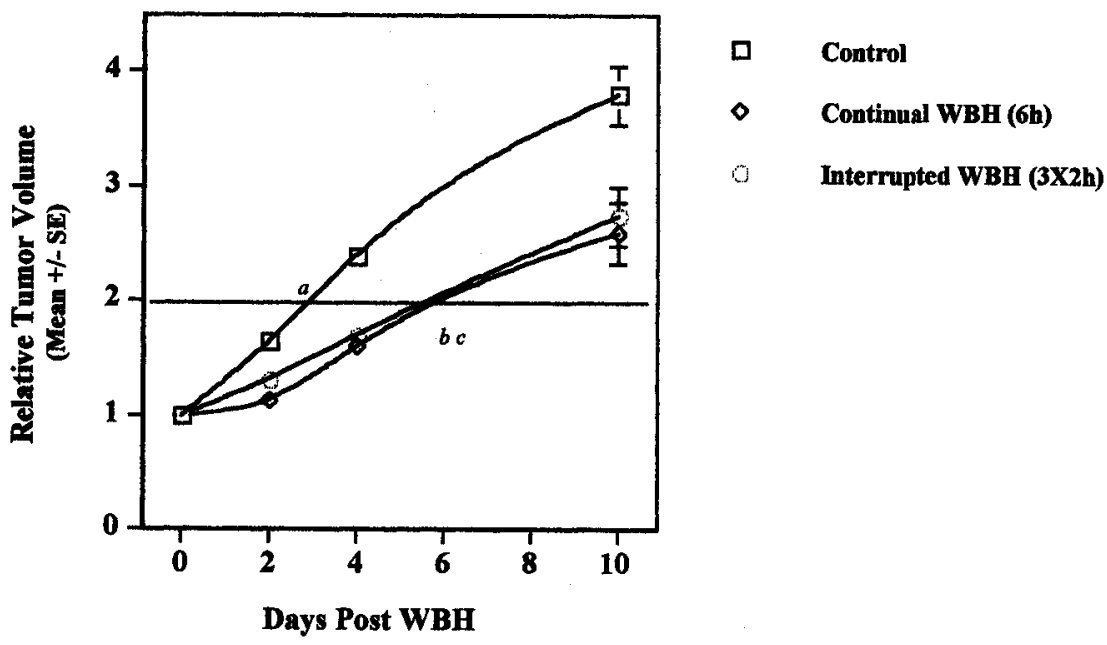

Fig. 4. Effect of continual and interrupted $\mathrm{WBH}$ on the growth of a human breast tumor $\# 8600$. SCID mice bearing a human breast tumor were treated with $\mathrm{WBH}$ on day 0 for 6 hours (continual $\mathrm{WBH}, \mathrm{n}=9$ ) or for 3 hours with a I5-minute break and continuing with another 3 hours for a total of 6 hours (interrupted $W B H, n=5$ ). In the control group (a), TGT was 3.0 \pm 0.01 d. In the continual WBH group (b), TGT was $7.3 \pm 0.8 \mathrm{~d}(P<0.01$ when compared with the control). In the interrupted WBH group (c), TGT was $6.2 \pm 1.0 \mathrm{~d}(P<0.01$ when compared with the control).

therapy or gene therapy, may prove to be even more successful.

Although combining WBH with other therapies is an ultimate goal, we are continually reexamining the effects of this model alone in order to obtain the greatest possible anti-tumor response. Thus far, our data has shown that 6 hours of $\mathrm{WBH}$ will give this maximum response. Interestingly, this treatment can be given in one long administration or in interrupted treatments. The interrupted WBH design could prove to be a more comfortable alternative to patients undergoing WBH. We have also shown that a second 6-hour WBH treatment appears to enhance the anti-tumor response observed with one treatment. However, for at least one model, a third WBH exhibited no apparent advantage over two treatments. Our laboratory is currently investigating possible reasons for this lack of efficacy. We speculate that thermotolerance may have a role; some permanent change in tumor structure may occur with the first and second treatments that result in the loss of response to more heat.

Current studies are also underway to demonstrate interdependence between the anti-tumor response seen with WBH and the changes our studies have shown on both lymphocytes and tumor cells. We have hypothesized that simple mild hyperthermia treatment may have the ability to activate antigen-independent pathways that result in enhanced lymphocyte polarity and adhesion. This in turn may facilitate subsequent antigen-dependent immune events. It is tempting to speculate that these same alterations may also occur during an actual fever following infection or other perturbations of the immune system. Since our data also indicate that even those mild WBH protocols can induce Hsps, we are very interested in determining what their role may be in stimulating the immune response.

A phase-I WBH clinical protocol has recently begun at Roswell Park Cancer Institute (under the direction of W. Kraybill, MD, Department of Surgery). It was designed to evaluate the safety and immunological effects of fever-like WBH alone in patients with advanced cancer. We will also continue to evaluate this fever-like WBH model preclinically in animal models. An understanding of the immune mechanisms that may be enhanced and their possible role in the anti-tumor response seen with WBH could lead to the development of novel therapies in the treatment of human breast, ovarian, and colon cancers.

\section{ACKNOWLEDGMENTS}

We would like to thank the Tissue Procurement Facility at Roswell Park Cancer Institute for providing us with all of the human tissue samples used in these experiments. We would also like to ac- 
knowledge Dr. Ning-Ping Yang for all of the time she spends looking after our mice.

\section{REFERENCES}

1. Burd R, Dziedzic 'TS, Xu Y, Caligiuri MA, Subjeck JR, Repasky EA. Tumor cell apoptosis, lymphocyte recruitment and tumor vascular changes are induced by low temperature, long duration (fever-like) whole body hyperthermia. J Cell Physiol 1998;177:137-147.

2. Di Y-P, Repasky EA, Subjeck JR. The distribution of HSP70, protein kinase $\mathrm{C}$ and spectrin is altered in lymphocytes during a fever-like hyperthermia exposure. J Cell Physiol 1997;172:44-54.

3. Matsuda H, Strebel FR, Kaneko T, et al. Long duration-mild whole body hyperthermia of up to 12 hours in rats: feasibility, and efficacy on primary tumour and axillary lymph node metastases of a mammary adenocarcinoma: implications for adjuvant therapy. Int J Hyperthermia 1997;13:89-98.

4. Engin K, Tupcheng L, Waterman FM, et al. Multiple field hyperthermia combined with radiotherapy in advanced carcinoma of the breast. Int J Hyperthermia 1994;10:587.

5. Lindholm CE, Kjellen E, Nilsson P, Weber L, Hill S. Prognostic factors for tumor responses and skin damage to combined radiotherapy and hyperthermia in superfi- cial recurrent breast carcinomas. Int J Hyperthermia 1995;11:337.

6. Oleson JR. Review: Eugene Robertson Special Lecture: Hyperthermia from the clinic to the laboratory: a hypothesis. Int J Hyperthermia 1995;11:315.

7. Pettigrew RT, Galt JM, Ludgate CM, Horn DB, Smith AN. Proceedings: The effect of whole body hyperthermia in advanced cancer. Brit J Cancer 1974;30:179.

8. Bull JM. Systemic hyperthermia: background and principles. In Storm FK (ed): Hyperthermia in Cancer Therapy. Boston: G.K. Hall Medical Publishers, 1983, pp 401-405.

9. Sakaguchi Y, Stephens LC, Makino M, et al. Apoptosis in tumors and normal tissues induced by whole body hyperthermia in cancer research. Cancer Res 1995;55: 5459-5464.

10. Robins HI, Kutz M, Wiedermann GJ, et al. Cytokine induction by $41.8^{\circ} \mathrm{C}$ whole body hyperthermia. Cancer Lett 1995;97:195-201.

11. Hughes CS, Repasky EA, Bankert RB, Johnson RJ, Subjeck JR. Effects of hyperthermia on spectrin expression patterns of murine lymphocytes. Radiat Res 1987; 112:116-121.

12. Wang X-Y, Ostberg JR, Repasky EA. Effect of feverlike whole-body hyperthermia on lymphocyte spectrin distribution, protein kinase $\mathrm{C}$ activity, and uropod formation. J Immunol. In press. 


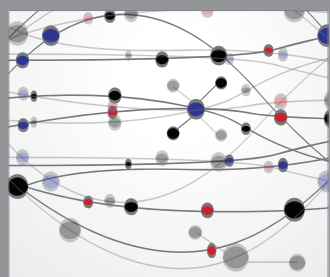

The Scientific World Journal
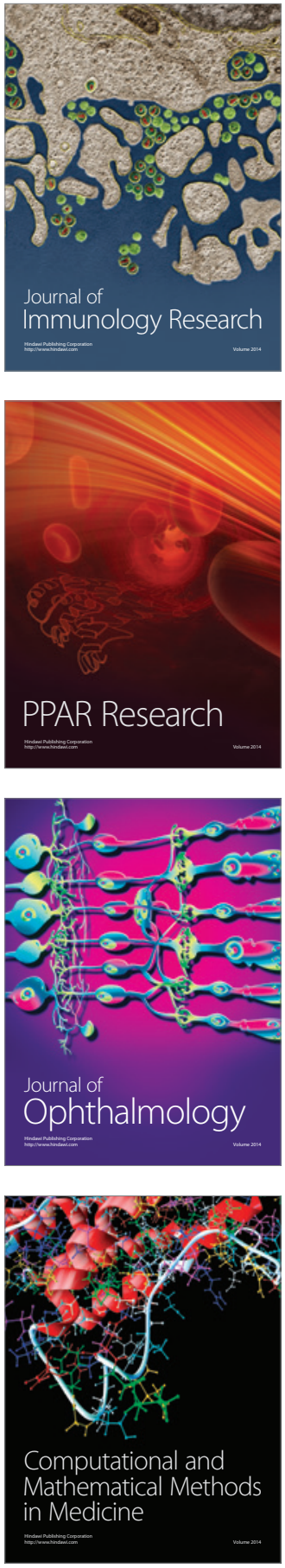

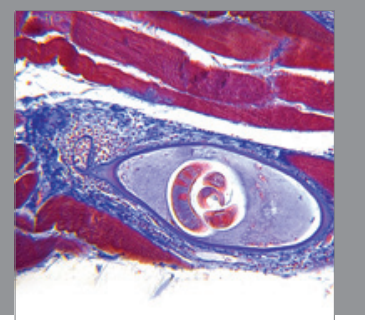

Gastroenterology

Research and Practice
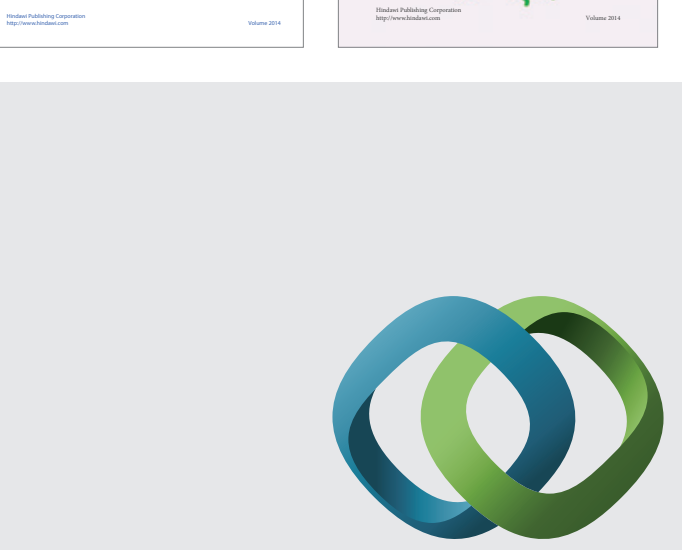

\section{Hindawi}

Submit your manuscripts at

http://www.hindawi.com
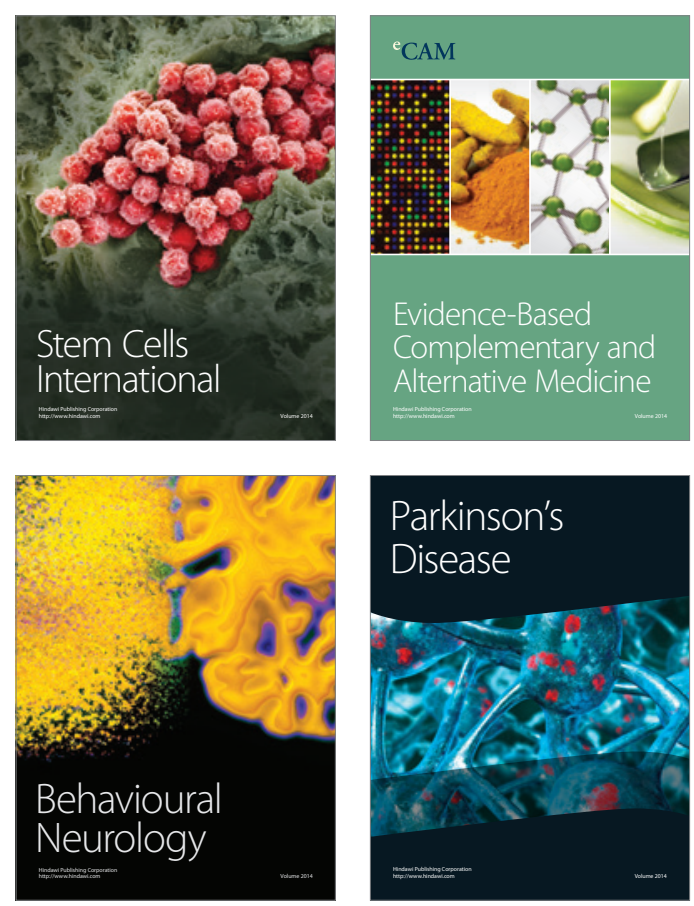

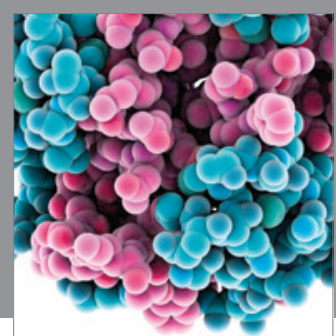

Journal of
Diabetes Research

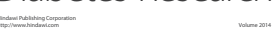

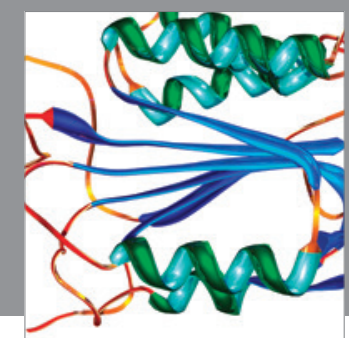

Disease Markers
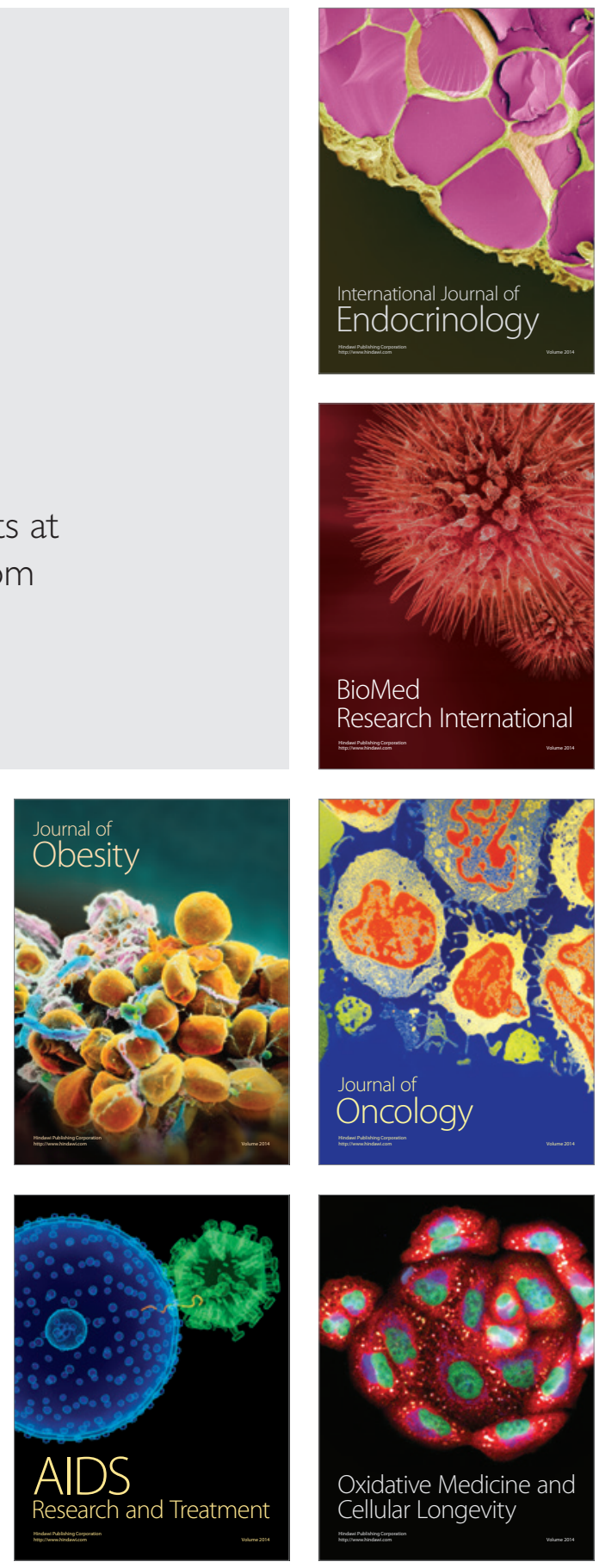